\title{
Diffusion of the atomic clusters over the (111) and (100) surfaces in Ni crystal
}

\author{
G. M. Poletaev ${ }^{1 \dagger}$, V. M. Kaygorodova ${ }^{1}$, G. A. Elli ${ }^{1}$, O. M. Uzhakina ${ }^{1}$, J. A. Baimova ${ }^{2}$ \\ †gmpoletaev@mail.ru
}

\author{
${ }^{1}$ Altai State Technical University, 46 Lenin st., 656038, Barnaul, Russia \\ ${ }^{2} \mathrm{~S} c h o o l$ of Mechanical and Aerospace Engineering, Nanyang Technological University \\ 50 Nanyang Avenue, 639798, Singapore, Singapore
}

\begin{abstract}
Diffusion of the atomic clusters over $\mathrm{Ni}(111)$ and (100) surfaces is studied by the molecular dynamics simulation. The activation energies of diffusion of the clusters of different sizes are calculated. It is shown, that cluster's atoms migrate over the surface by individual movement instead of collective motion.
\end{abstract}

Keywords: molecular dynamics, surface diffusion, cluster, diffusion activation energy, migration mechanisms.

\section{Introduction}

Surface diffusion is a movement of the adsorbed particles such as atoms or molecules on the metal's surface. Activation energy of the surface diffusion is one order of magnitude less than activation energy of diffusion in the bulk [1,2] which is the reason for surface diffusion to be much more intense. Surface diffusion plays determining role in the processes of nanostructure formation on the substrate surface. Investigation of the surface diffusion is very important for the issues of catalysis, adsorption and film growth.

During the migration over the surface, separated atoms can approach each other and, in the presence of mutual interaction, form stable cluster which contains from two atoms to several hundreds of atoms. Two aspects of the cluster dynamics can be taken into account: redistribution of the atoms in the cluster and diffusion of the cluster as a whole. Surface diffusion of the cluster can be characterized by the displacement of the cluster mass center. In this regard, migration of the cluster can also be considered as the migration of single adatom. Numerous experimental observation show, that usually, the greater the cluster, the less is its mobility [1].

Existing experimental methods cannot directly define the leading mechanism of cluster migration over the substrate surface. Such methods as field ion microscopy and scanning tunnel microscopy enable to get the «snapshot» of the cluster before and after diffusion event, but generally cannot show the short-lived transition state. Therefore, diffusion mechanisms are usually determined from the indirect data such as activation barrier value, dependence of the cluster mobility from its size, cluster shape modification during diffusion. Computer simulation is one of the most effective methods for these studies. For example, molecular dynamics (MD) simulation was successfully used for the investigation of plastic deformation of polycrystals on atomistic level, studying of defect migration, energy distribution, ets. [3-6].

The goal of the present work is the investigation of the cluster diffusion over $\mathrm{Ni}(111)$ and (100) surfaces by means of MD simulation.

\section{Simulation details}

Computational cell has the form of parallelepiped, composed of seven atomic planes (Fig.1). Two lower atomic layers are rigidly fixed with all the atoms preserved immobile during the numerical experiments, to hold the crystallographic orientation of the simulated surface unchanged. Two different types of surface packing corresponding to (111) and (100) crystallographic planes of $f c c$ crystal are considered. On the opposite side of the rigidly fixed atomic planes the free surface is simulated. Periodic boundary conditions, imitating endless repetition of the considered region along $\mathrm{X}$

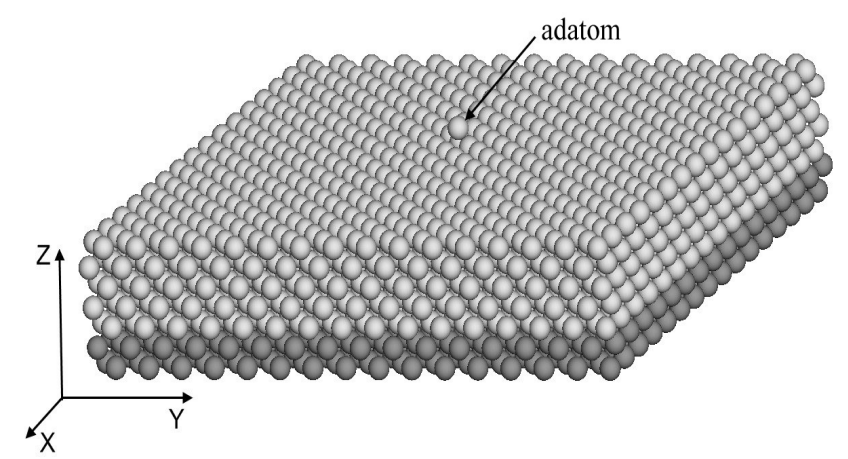

Fig. 1. Simulation cell, containing the region of (111) surface with adatom. Atoms shown in dark-gray stay immobile during numerical experiments. Periodic boundary conditions are applied along $\mathrm{X}$ and $\mathrm{Y}$ axes. 
and $\mathrm{Y}$ directions, are applied to the lateral surfaces.

Number of atoms at $0 \mathrm{~K}$ is about 4000 atoms with the total size of the computational cell $52 \times 52 \times 14 \AA$ in case of (111) surface, and 56x56x13 $\AA$ in case of (100) surface.

Cluster with the number of atoms ranging from one to seven is placed on the simulated surface (Fig.2). The shape of the clusters, shown in Fig. 2 corresponds to the minimal potential energy. In course of the migration in molecular dynamics simulation, clusters tend to preserve its shape.

The relaxation of the structure (MD experiment at the initial temperature $0 \mathrm{~K}$ ) is carried out. Atoms on the surface are taking the low-energy positions during relaxation, and the temperature of the simulation cell is slightly increased. Finally, the relaxed simulation cell is cooled.

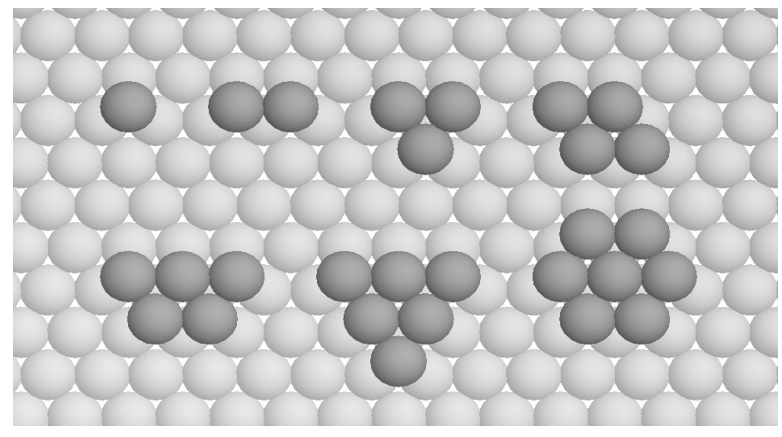

a

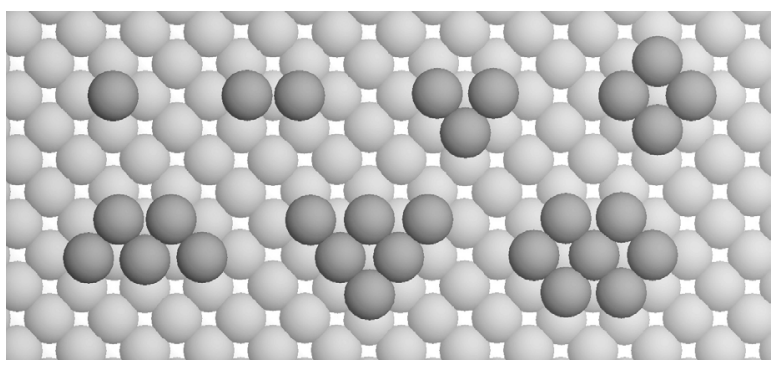

b

Fig. 2. Clusters, containing different number of atoms from 1 to 7 , on the $\mathrm{Ni}(111)$ (a) and (100) (b) surfaces.

Many-body Cleri-Rosato potential, obtained in the frames of strong interaction model, is used to describe interatomic interactions [7]. This potential was successfully used in the various applications, including the calculation of energy and diffusion characteristics with the help of MD simulations $[8,9]$. The cut off radius of the potential is 5 coordination spheres. Simulation time step is equal to $10^{-14} \mathrm{~s}$. In $[10,11]$ it was shown that this time step is quite enough for the simulation of thermal movement of $\mathrm{Ni}$ atoms.

At present work, simulation of cluster migration is considered at different temperatures. The temperature is introduced by the setting of the initial velocities of the atoms in accordance with the Maxwell distribution. Interatomic distances in this case were corrected in accordance with thermal expansion.

To define the coefficients of surface self-diffusion, the numerical experiments during 0.1-0.2 ns are carried out at the constant temperature (simulation of the thermostat). This time is quite enough because the surface diffusion is a fast process and enough number of atomic displacements takes place during this time.
Temperature range from $500 \mathrm{~K}$ to $1700 \mathrm{~K}$ is investigated. At the final stage of the numerical experiment the cooling of the computational cell to $0 \mathrm{~K}$ took place, to exclude the thermal displacements of atoms. Diffusion coefficient along the $\mathrm{X}$ axis is calculated as follows

$$
D_{x}=\frac{\frac{1}{N} \sum_{i=1}^{N}\left(x_{0 i}-x_{i}\right)^{2}}{2 t},
$$

where $x_{0 \mathrm{i}}-$ coordinate of the initial position of $i$-th atom; $x_{\mathrm{i}}$ - coordinate of $i$-th atom at the moment $t ; N-$ number of atoms in the computational cell. Diffusion coefficient along Y axis is calculated in the same way. The average self-diffusion coefficient $D$ is the arithmetical mean of $D_{\mathrm{x}}$ and $D_{\mathrm{y}}$.

Activation energy of migration of atom clusters over the surface is calculated from the temperature dependence of the diffusion coefficient [8]. For each cluster the dependence $\ln D\left(T^{-1}\right)$ is plotted. Activation energy $E_{\mathrm{m}}$ in this case can be defined from the slope of the curve for $\alpha: E_{\mathrm{m}}=-k \cdot \operatorname{tg} \alpha$, where $k$ is the Boltsman constant.

To study the migration mechanisms of atom clusters over the surface, the visualizer of atomic displacements showing the atomic displacements from initial to final configurations during numerical experiment is used [12-15].

\section{Results and discussion}

In Table 1, the values of activation energies of cluster migration over (111) and (100) surface as the function of number of atoms in the cluster are presented. It should be noted, that the observed values are significantly less than activation energy of diffusion in the bulk. For example, migration energy of the vacancy in $\mathrm{Ni}$ from various studies $[16,17]$ changing from 0.9 to $1.5 \mathrm{eV}(0.97 \mathrm{eV}$ - in accordance with our calculations by means of MD [9]). This result is in a good agreement with the results of other authors. Activation migration energies of the atomic clusters over the surface presented in this work are considerably lower than the activation migration energy of grain boundary diffusion: $1-1,5 \mathrm{eV}[14,15,18-20]$

The average value of the activation energy of the surface diffusion for $\mathrm{Ni}$ is equal to $0.91 \mathrm{eV}$ [2]. It is much higher that one obtained in the model and close to the vacancy migration energy. It should be taken into account, that in this value besides the energy of the migration by the different

Table 1.

Activation energy of the migration of cluster over the surface of the $\mathrm{Ni}$ crystal as the function of the number of atoms in the cluster.

\begin{tabular}{|c|c|c|}
\hline $\begin{array}{c}\text { Number of atoms } \\
\text { in the cluster }\end{array}$ & Surface (100) & Surface (111) \\
\hline 1 & 0.42 & 0.11 \\
\hline 2 & 0.53 & 0.19 \\
\hline 3 & 0.56 & 0.22 \\
\hline 4 & 0.63 & 0.23 \\
\hline 5 & 0.65 & 0.25 \\
\hline 6 & 0.68 & 0.27 \\
\hline 7 & 0.71 & 0.28 \\
\hline
\end{tabular}


mechanisms (different migration mechanisms contributing to the surface diffusion are: migration of adatoms and atom clusters over the plane surfaces, evaporation and condensation, ets.), the energy of the formation of the adatom or cluster of atoms on the surface is included.

From Table1 it can be seen that with the increase of the cluster size, the energy of cluster migration is increased, i.e. its mobility is decreased. This result is quite logic and in a good agreement with the results of other authors [1,2]. In Ref.[1], it is shown that the decrease of the mobility with the increase of cluster size is a general tendency. However, significant deviation can take place in some systems, when the cluster shape affects its mobility among the other factors. For instance, the oscillating character of the dependence of the diffusion coefficient from the cluster size was observed for Rh clusters on the Rh (100) surface. In this system, the higher activation energies were found for the clusters of more compact shape.

As it can be seen from Table 1, migration activation energies of the cluster over the (111) surface are considerably lower than that for (100) surface. In other words, the potential wells, in which the surface atoms are placed, deeper in case of (100) surface, in comparison with the relatively smooth (111) surface.

In case of surface diffusion, the diffusion of single adatom should be separated from the cluster diffusion. Substantially, there are two main mechanisms of adatom migration [1,2]: hopping and exchange diffusion. Hopping mechanism is the thermally activated jump of the adatom from one equilibrium position to another. It was shown $[1,2]$ that despite its simplicity, this mechanism gives right description of the surface diffusion in the realistic systems. The second mechanism is the swapping of adatom and the surface atom. In this case, adatom substitute the substrate atom and the surface atom moves to the neighboring adatom position. In some cases, this mechanism is energetically more preferable than the simple hopping of the adatom over the potential barrier, because during this exchange all the atoms preserve the majority of its bonds with the neighboring atoms.

Diffusion mechanism becomes more complicated when the diffusing of the whole clusters is considered instead of diffusion of single adatom. Depending on the specified conditions, atomic clusters can move very differently that is why a lot of various migration mechanisms over the surface have been proposed nowadays. All the basic mechanisms can be divided into two main types [1]: individual (based on the movement of the single atom) and collective mechanisms (based on the simultaneous movement of the group of atoms).

Individual mechanisms are related to the cases when the movement of the whole cluster is the result of the independent movement of the atoms constituting the cluster. Among them are $[1,2]$ : mechanism of consistent displacements (movement of the separated atoms one from another); mechanism of edge (or peripheral) diffusion when the displacement of the edge adatoms, vacansies and the breaks on the edge of the cluster leads to the displacement of cluster mass center; mechanism of evaporation-condencation (diffusion because of the exchange between cluster atoms and atoms of twodimensional adatom gas, i.e. the evaporation is considered as the separation of atoms from the cluster); mechanism of the "reshuffle" when one of the cluster edge atoms goes over the cluster and join it from the opposite side.

Collective mechanisms describe the situation when the displacements of the cluster took place because of simultaneous correlated displacement of at least the part of the cluster. The following mechanisms can be distinguished $[1,2]$ : sliding mechanism related to the sliding of the cluster as the whole; sliding mechanism, in which the group of atoms (for example, the row of atoms in the cluster) perform the concerted motion (shear); climbing mechanism which resides in the sequential shear of the neighboring cluster parts which leads to the serpentine sliding movement.

In present work, the investigation of the leading mechanism is carried out with the help of atomic displacements visualizer. In course of the numerical experiment, where the migration of the cluster over the surface took place, the visualizer demonstrates atomic displacements by the segments connecting initial and current atomic positions.

In Fig.3, the example of the adatom displacement over the (100) surface at $1200 \mathrm{~K}$ is shown. It can be seen, that adatom migrates by the hopping mechanism, i.e. no exchange of the adatom and substrate atoms took place (exchange mechanism is not realized).

Atoms on the (100) surface are placed in the relatively deep potential wells in comparison with (111) surface. Energy barrier, required for the jump to the neighboring well on the

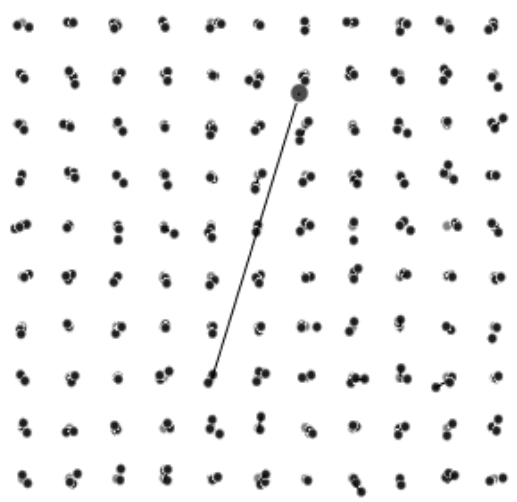

Fig. 3. Displacement of the adatom in coarse of migration over $\mathrm{Ni}$ (100) surface at $1200 \mathrm{~K}$ during $5 \mathrm{ps}$. Adatom is shown by big gray circle.

(100) surface is higher than for (111) surface which can be seen from Table 1. Thereby, the exchange mechanism should be realized more frequently for the (100) surface, however, the exchange between atoms on the surface (adatoms) and substrate atoms is very seldom and the migration occur, generally, by hopping mechanism.

In Fig. 4 the examples of the atomic displacements during the migration of atomic cluster composed of 3 atoms over (111) and (100) surfaces is presented.

It should be noted that the cluster migration, as it can be seen from Fig.4, proceeds by the individual mechanism. Cooperative mechanisms are not realized - atomic displacements are not consistent, the frequent crosses took place and atoms randomly change their positions in the cluster. Nevertheless, the cluster in the most cases (at not very high temperatures) stay stable and no destruction is observed, as it is shown in Fig.4a. With the increase in temperature the possibility of cluster destruction increases, and at the high 


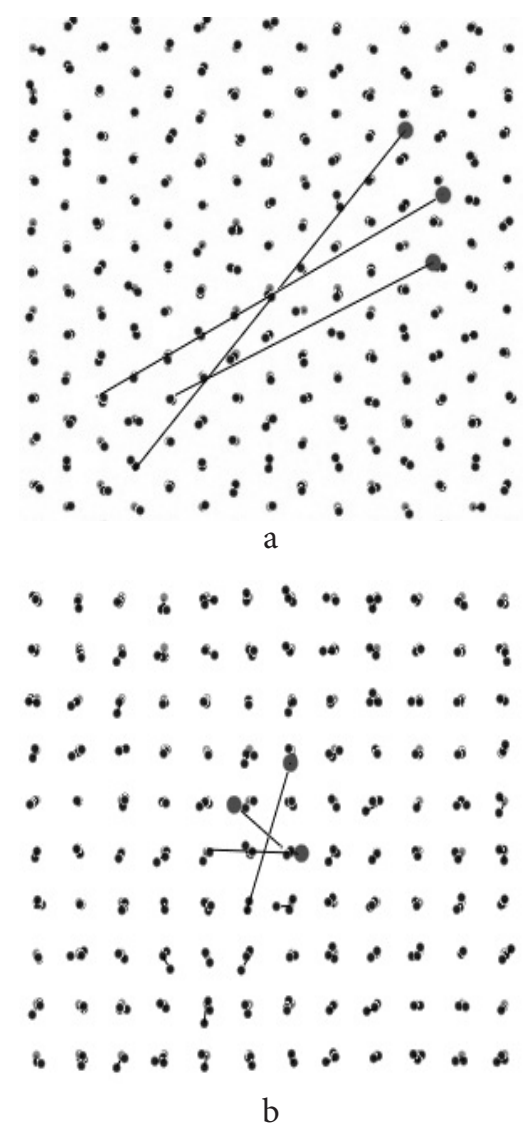

Fig. 4. Atomic displacements in coarse of migration of the cluster composed of 3 atoms over the (111) (a) and (100) (b) surface at $1000 \mathrm{~K}$ during $5 \mathrm{ps}$. Atoms on the surface are shown by bigger circles.

temperatures the cluster destruction is quite often event.

The lack of consistency during cluster migration can be clearly seen not only from the atomic displacement's visualizer but also from the direct observation of the atomic movement in the numerical experiment. From these observations, it is shown that basically individual mechanism of the edge (or peripheral) diffusion is realized, i.e. atoms on the cluster's edge are more mobile and placed in the shallower potential wells, and the migration of the whole cluster is connected with the mobility of such atoms.

\section{Conclusion}

Thereby, at present work the values of the migration activation energies of the clusters of different size over the $\mathrm{Ni}$ (111) and (100) surfaces are calculated by means of MD simulation. It was shown that with the increase of the cluster size its mobility decreases, while the migration energy is increased. Clusters on the (111) surface are considerably more mobile in comparison with the clusters on (100) surface.

Migration of the single atom (adatom) over Ni surface is realized by the hopping mechanism, and no exchange between the substrate atoms and cluster atoms during the adatom migration is observed (i.e. the exchange mechanism is not realised). As it is shown, atoms in the cluster migrate predominantly by the individual movement instead of collective motion.
Authors are grateful acknowledge the financial support from Russian Foundation for Basic Research grants №13-0200301_a, № 14-02-98000-r_sibir_a, № 14-08-90416-Ukr_a.

\section{References}

1. K. Oura, V.G. Lifshits, A.A. Saranin, A.V. Zotov, M. Katayama. Introduction to the surface physics. Moscow, Nauka. (2006) 490 p. (in Russian)

2. M. Pratton. Introduction to the surface physics, Izhevsk, SRC "Regular and chaotic dynamics". (2000) 256 p. (in Russian)

3. E.A. Korznikova, J.A. Baimova, A.A. Kistanov, S.V. Dmitriev, A.V. Korznikov. Fizika metallov i metallovedenie. 115(9), 977 (2014). (in Russian)

4. E. A. Korznikova. Letters on Materials. 3(4), 330 (2013).

5. A.A. Nazarova, S. V. Dmitriev, Yu.A. Baimova, R. R. Mulyukov, A.A. Nazarov. The Physics of Metals and Metallography. 111(5), 513 (2011).

6. J.A. Baimova, S. V. Dmitriev. Physical Mesomechanics. 13 (6), 53 (2010).

7. F. Cleri, V. Rosato. Physical Review. B48(1), 22 (1993).

8. G. M. Poletaev, M.D. Starostenkov. Physics of the Solid State. 52 (6) 1146 (2010).

9. G. M. Poletaev, D. V. Dmitrienko, M. D. Starostenkov. Fundamentalnye problemy sovremennogo materialovedenia. 9(3), 344 (2012). (in Russian)

10. A.A. Valuev, G.E. Normann, V.Yu. Podlipchuk. In Mathematical modelling: Physical-chemical properties of the matter. M. Nauka. (1989) p. 5-40. (in Russian)

11. M.D. Starostenkov, N. N. Medvedev, G. M. Poletaev. Izmerenie, avtomatizatsiya I modelirovanie $\mathrm{v}$ promishlennosti i tochnih issledovaniyah; interuniversity collection. AltGTU. (2005) p. 5-8. (in Russian)

12. E. G. Kharina, M.D. Starostenkov, G. M. Poletaev, R. Y. Rakitin. Physics of the Solid State. 53(5), 1043 (2011).

13. N. A. Kulabukhova, G. M. Poletaev, M. D. Starostenkov. Fundamentalnye problemy sovremennogo materialovedenia. 11(2), 235 (2014). (in Russian)

14. R. Yu. Rakitin, G.M. Poletaev, M.S. Aksenov, M.D. Starostenkov. Fundamentalnie problemi sovremennogo materialovedeniya. 2, 124 (2005). (in Russian)

15. A.N. Martinov, G. M. Poletaev, M.D. Starostenkov. Letters on Materials. 1(1), 43 (2011). (in Russian)

16. G. Y. Vollenberg. Point defects. In Physics of material science. V. 3. Physical and mechanical properties of metals and alloys. M. Mir. (1987) p. 5-74. (in Russian)

17. A. N. Orlov, Yu. V. Trushin. Point Defect Energies in Metals. M.:Energoatomisdat. (1983) 80 p. (in Russian)

18. R. T. Murzaev, A. A. Nazarov. The Physics of Metals and Metallography. 100(3), 228 (2005).

19. R. T. Murzaev, A. A. Nazarov. The Physics of Metals and Metallography. 101(1), 86 (2006).

20. R. T. Murzaev, A.A. Nazarov. The Physics of Metals and Metallography. 102(2), 198 (2006). 